\title{
Are some individuals generally more behaviorally plastic than others? An experiment with sailfin mollies
}

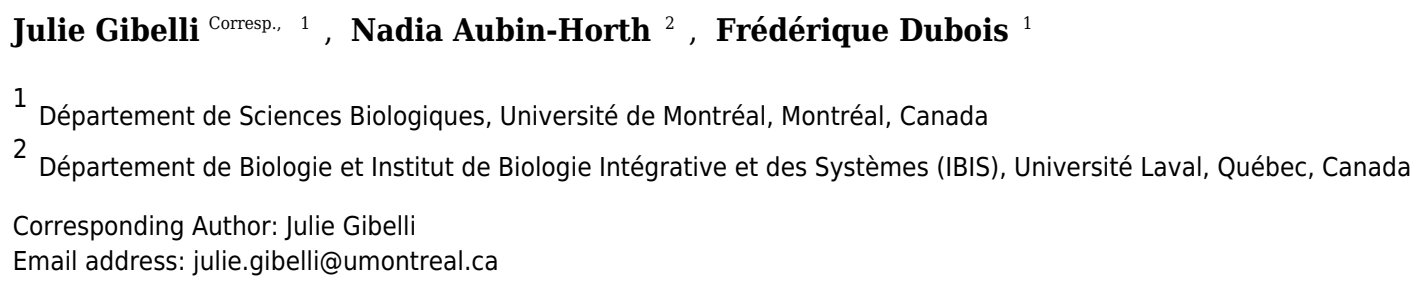

Individuals within the same population generally differ among each other not only in their behavioral traits but also in their level of behavioral plasticity (i.e. in their propensity to modify their behavior in response to changing conditions). If the proximate factors underlying individual differences in behavioral plasticity were the same for any measure of plasticity, as commonly assumed, one would expect plasticity to be repeatable across behaviors and contexts. Yet, this assumption remains largely untested. Here we conducted an experiment with sailfin mollies (Poecilia latipinna) whose behavioral plasticity was estimated both as the change in their personality traits or mating behavior across a social gradient and using their performance on a reversal-learning task. We found that the correlations between pairwise measures of plasticity were weak and non-significant, thus indicating that the most plastic individuals were not the same in all the tests. This finding might arise because either individuals adjust the magnitude of their behavioral responses depending on the benefits of plasticity, and/or individuals expressing high behavioral plasticity in one context are limited by neural and/or physiological constraints in the amount of plasticity they can express in other contexts. Because the repeatability of behavioral plasticity may have important evolutionary consequences, additional studies are needed to assess the importance of trade-offs between conflicting selection pressures on the maintenance of intra-individual variation in behavioral plasticity. 
$1 \quad$ Are some individuals generally more behaviorally plastic than others?

2

\section{An experiment with sailfin mollies}

3

4

5

Julie Gibelli ${ }^{1}$, Nadia Aubin-Horth ${ }^{2}$ and Frédérique Dubois ${ }^{1}$

1 Département de Sciences Biologiques, Université de Montréal, Montréal, Canada

62 Département de Biologie et Institut de Biologie Intégrative et des Systèmes (IBIS), Université

7

Laval, Québec, Canada

8

9 Corresponding author:

10 Julie Gibelli

11 Email address: julie.gibelli@umontreal.ca 


\section{ABSTRACT}

14 Individuals within the same population generally differ among each other not only in their

15 behavioral traits but also in their level of behavioral plasticity (i.e. in their propensity to modify 16 their behavior in response to changing conditions). If the proximate factors underlying individual 17 differences in behavioral plasticity were the same for any measure of plasticity, as commonly 18 assumed, one would expect plasticity to be repeatable across behaviors and contexts. Yet, this 19 assumption remains largely untested. Here we conducted an experiment with sailfin mollies 20 (Poecilia latipinna) whose behavioral plasticity was estimated both as the change in their 21 personality traits or mating behavior across a social gradient and using their performance on a 22 reversal-learning task. We found that the correlations between pairwise measures of plasticity 23 were weak and non-significant, thus indicating that the most plastic individuals were not the 24 same in all the tests. This finding might arise because either individuals adjust the magnitude of 25 their behavioral responses depending on the benefits of plasticity, and/or individuals expressing 26 high behavioral plasticity in one context are limited by neural and/or physiological constraints in 27 the amount of plasticity they can express in other contexts. Because the repeatability of 28 behavioral plasticity may have important evolutionary consequences, additional studies are 29 needed to assess the importance of trade-offs between conflicting selection pressures on the 30 maintenance of intra-individual variation in behavioral plasticity. 


\section{INTRODUCTION}

33 Over the past decades, considerable interest has been devoted to the study of animal personality,

34 which is defined as a suite of individual differences in behavior that are consistent over time and 35 contexts (Sih \& Bell, 2008). Numerous studies on animal personality, notably, have 36 demonstrated that individuals within the same population generally differ widely among each 37 other not only in their behavioral traits such as their activity level, exploration tendency or 38 aggressiveness, but also in their propensity to modify their behavior in response to changing 39 conditions (that is in their level of behavioral plasticity, also referred to as contextual plasticity, 40 41 42 43 activational plasticity, reversible plasticity or responsiveness; e.g. Stamps, 2016). Behavioral plasticity is often considered advantageous (DeWitt, Sih \& Wilson, 1998; Gabriel et al., 2005). Accordingly, comparative analyses have shown that bird species with relatively large brains and 3 a high frequency of foraging innovations tend to be more successful invaders than less flexible species (Sol \& Lefebvre, 2000; Sol, Timmermans \& Lefebvre, 2002). Also, at the intraspecific

level, two recent empirical studies found that individuals with greater behavioral plasticity had higher fitness, in terms of survival (Toscano, 2017) or mating success (Montiglio et al., 2017), 48 compared to less plastic ones. If being plastic is advantageous, however, natural selection should erode variations in behavioral plasticity and several evolutionary explanations have thus been proposed to account for the maintenance of individual differences (Wolf, van Doorn \& Weissing, 2008; Dubois, Morand-Ferron \& Giraldeau, 2010; Wolf, Van Doorn \& Weissing, 2011). Although those explanations rely on different processes (e.g. state-dependent selection, frequency-dependent selection, positive feedback loops), they all implicitly assume that the proximate factors underlying individual differences in behavioral plasticity are the same for any measure of plasticity, and that an individual's plasticity, therefore, should be repeatable across behaviors and contexts (Dingemanse \& Wolf, 2013).

In particular, in order to adjust its behavior to local conditions, an animal must detect and respond to environmental changes. Personality-related differences in behavioral plasticity could then result from individual differences in sampling behavior, sensitivity to external stimuli, risktaking tendency or cognitive ability (Carere \& Locurto, 2011; Sih \& Giudice, 2012). Accordingly, several studies have shown that individual differences in behavioral plasticity or purported measures of plasticity such as reversal learning rate (e.g. Guillette et al., 2010; Lucon- 
63 Xiccato \& Bisazza, 2014; Pintor et al., 2014) are associated with differences in personality traits 64 (e.g. Gibelli \& Dubois 2017; Guayasamin, Couzin \& Miller, 2017; Guido et al. 2017; Mazza et 65 al. 2018; see also reviews by Mathot et al., 2012 and Stamps, 2016), hence suggesting that 66 certain behavioral traits or particular skills that are associated with them could prevent 67 individuals from exhiting optimal behavioral plasticity. By contrast, however, several authors 68 reported the opposite effect or no effect of personality type on how individuals respond to 69 changing stimuli (e.g. Logan 2016b; Bensky et al. 2017), whereas the few studies that have 70 measured behavioral plasticity for the same individuals in several traits or contexts (Biro, 71 Beckmann \& Stamps, 2010; Morand-Ferron, Varennes \& Giraldeau, 2011; Logan 2016a) or 72 from different proxies (Brucks et al. 2017; Johnson-Ulrich et al. 2018) found weak or no support 73 for a general plasticity. For instance, despite most individuals becoming more active and bold as 74 temperature increased in damselfish (Pomacentrus bankanensis), Biro et al. (2010) found that the 75 degree of plasticity in activity of a given individual was unrelated to its level of plasticity in 76 boldness. In the same vein, Morand-Ferron et al. (2011) found that the most plastic individuals 77 were not the same in two foraging games in nutmeg mannikins (Lonchura punctulata) while Mitchell \& Biro (2017) reported that zebrafish (Danio rerio) that displayed high responsiveness to temperature were not more responsive to food deprivation. A lack of correlation in individual 81 plasticity across contexts or behaviors could be explained by at least the two following causes.

First, behavioral plasticity would not be a general feature of an individual because its ability to perceive changes in environmental conditions and adjust its behavior accordingly, is affected by the magnitude of the changes. More precisely, the speed-accuracy trade-off hypothesis (Chittka, Skorupski \& Raine, 2009) predicts that fast-exploring individuals should make faster but less accurate decisions compared to slow-exploring individuals that invest more time in decision process. Such a trade-off between speed and accuracy, however, would only exist when the variations in environmental conditions are subtle and hence require considerable sampling effort to be detected. Conversely, when changes in external stimuli are easy to detect, fast-exploring individuals might be capable of making fast and accurate decisions (Mamuneas et al., 2015). According to this idea, an individual that explores its environment superficially would then be unlikely to adjust its behavior to local conditions when the changes in environmental conditions 
94 low individual consistency in plasticity across behaviors could result from physiological 95 constraints that would reduce the capacity of individuals to adjust hormonally-mediated 96 behaviors to changing environmental conditions (Hau \& Goymann, 2015). For instance, 97 experimentally elevated levels of testosterone have been shown to increase courtship behavior 98 and aggression but to reduce parental care in several species (McGlothlin, Jawor \& Ketterson, 99 2007), hence suggesting that its physiological state could, in some cases, prevent an individual to 100 respond optimally to changing environmental conditions. Whether the level of behavioral 101 plasticity expressed by an individual is limited by its ability to detect environmental changes or 102 by its physiological state, we would then expect individual differences in behavioral plasticity to 103 be associated with individual differences in personality traits. However, the strength and 104 direction of the correlations should vary depending on the measure of plasticity and/or on the 105 magnitude of the change in environmental conditions.

106

107 In order to test whether some individuals are generally more behaviorally plastic than others, as 108 commonly assumed, or if their ability to adjust their behavior in response to changing conditions 109 is trait and/or context-dependent, we conducted an experiment with sailfin mollies (Poecilia 110 latipinna). For each fish, we measured its behavioral plasticity both as the change in its 111 personality traits or mating behavior in response to changes in environmental stimuli and as its 112 performance on a reversal-learning task. Then, we tested whether the different measures of 113 behavioral plasticity were correlated between each other.

114

\section{MATERIALS \& METHODS}

\section{Animals and housing conditions}

117 We used 57 (41 males and 16 females) sexually mature sailfin mollies aged 4 months that had 118 not participated in any previous experiment: 33 males were used as focal individuals whereas 8 119 other males and 16 females served as audience individuals. All individuals came from a 120 commercial fish supplier (Mirdo Importations Canada Inc, Montreal, Quebec, Canada). The fish 121 were kept in brackish water at $24 \pm 0.5^{\circ} \mathrm{C}$ with a $12: 12$ photoperiod and were fed with spirulina 122 flakes (2\% of their weight) and brine shrimps twice a day. Outside the experiments, focal males

123 were housed either individually (15 individuals) in $3 \mathrm{~L}$ tanks $(10 \times 20 \times 15 \mathrm{~cm})$ or socially at a 124 density of $3 \pm 1$ fish (18 individuals) in $6 \mathrm{~L}$ tanks $(20 \times 20 \times 15 \mathrm{~cm})$, whereas the other fish that 
125 served as audience individuals were housed in $6 \mathrm{~L}$ tanks with a maximum of 3 or 5 same-sex

126 congeners, for males and females, respectively. The experiments were conducted in June 2015 at

127 the LARSEM (Laboratoire aquatique de recherche en sciences environnementales et médicales,

128 Université Laval, Québec, Qc, Canada), were approved by the Animal Care Committee of the

129 Université Laval (animal care permit \#2015027-1) and conformed to all guidelines of the

130 Canadian Council on Animal Care.

131

132 Male body length

133 The week before the beginning of the experiments, all male sailfin mollies were tranquilized

134 with a low dose (5 mg L) of anaesthetic (TMS-222), tagged with an elastomer tag (Northwest

135 Marine Technologies, Shaw Island, WA, USA) and photographed. Body length (distance from

136 the tip of the snout to the base of the tail fin) was then measured (precision $0.1 \mathrm{~mm}$ ) using Adobe

137 Photoshop CC. The measurements were taken 3 times on each individual by the same examiner

138 and then averaged. Based on the distribution of male body length, we then defined 3 categories

139 of males: small $(<5.90 \mathrm{~cm})$, medium $(5.90$ to $6.56 \mathrm{~cm})$ and large $(>6.56 \mathrm{~cm})$.

140

\section{Measures of plasticity}

142 All focal males were then tested in 3 experiments (i.e. mating behavior, personality and reversal 143 learning) in the same order. The experiments were performed between 8:30 am and 3:30 pm. All 144 trials were conducted in a $54 \mathrm{~L}$ tank $(601 \times 30 \mathrm{w} \times 30 \mathrm{~h} \mathrm{~cm})$ whose sides were covered by black 145 cloth in order to minimize disturbance and were recorded with a digital camera (JVC model GZ146 MS120).

147

148 1. Mating behavior

149 As in Fraser et al. (2014), we quantified the mating behavior of each focal male first in a control 150 condition (i.e. with 3 females) and then in a competitive condition (i.e. in presence of 3 male 151 competitors and 6 females), with a one-week delay between the two conditions. For the 152 competitive condition, we insured that the 3 males were of different sizes (i.e. one small, one 153 medium and one large fish) and that the size difference between 2 males was larger than $0.6 \mathrm{~cm}$. 154 Before being tested, the focal fish were isolated from their conspecifics for $24 \mathrm{~h}$. For each test, 155 we successively introduced into the tank the audience females, the audience males, when 
156 applicable, and finally the focal male. Then, during a 10-min observation period, we scored the 157 male's sexual behavior as 1) the number of nibbles in the vicinity of a female's urogenital 158 opening and 2) the number of gonoporal thrusts, as both traits have been shown to be plastic in 159 response to changes in competitive conditions (Travis \& Woodward, 1989; Fraser et al. 2014). 160 After the tests were completed, we calculated the plasticity in mating behavior of each male as 161 the difference in the number of nibbling bites or gonoporal thrusts between the control and 162 competitive conditions both in absolute and relative values. For subsequent analysis, however, 163 we only used plasticity in the number of nibbling bites as only this trait was significantly affected 164 by the condition though both traits were correlated (supplementary material 1). Given that the 165 males were housed with a number of conspecifics that varied between zero and 4 before being 166 isolated for $24 \mathrm{~h}$ and then tested, we insured that the housing condition of the fish had no effect 167 on their mating behaviors (supplementary material 1).

168

\section{2. Personality}

170 We conducted 2 behavioral tests that are commonly used to assess personality traits (Réale et al., 171 2007). Specifically, the open-field test was used to measure exploration and thigmotaxis, an 172 indicator of anxiety (Maximino et al. 2010), while the novel object test was used to measure 173 neophobia. All individuals performed the 2 tests in the same order (i.e. open field test and novel 174 object test) and each test was replicated twice without an audience (T1, T2) and twice with a 175 randomly selected audience (T3, T4). For each test, the interval between the alone and audience 176 treatments was 7 days, while the delay between 2 replicates for a given treatment varied between 1776 and $24 \mathrm{~h}$.

178

179 Open-field test: Exploration and anxiety

180 Prior to testing, the fish were familiarized with the tank for $30 \mathrm{~min}$ twice a day for 7 consecutive 181 days. The tank was equipped with 4 transparent plastic containers ballasted with 4-5 sterilized 182 black rocks, one in each corner, where we could confine the audience individuals (Fig. 1). 183 Specifically, for the audience treatment, 4 individuals (i.e. 2 males and 2 females) were placed in 184 the containers (i.e. one individual per container) before the focal fish was introduced, while the 185 containers were kept empty for the alone treatment. The containers were closed at the top with a 186 net, thereby preventing physical, but not chemical, interactions. At the beginning of a trial, we 
187 first introduced the focal fish in the center of the tank and then, once we had removed the landing 188 net from the top of the tank, we monitored its movements for 5 min using a custom software. The 189 images were divided into squares of $35 \times 35$ pixels (around $5 \times 5 \mathrm{~cm}$ ), so that we could estimate,

190 for each focal fish, its exploration tendency, as the number of different squares visited during the

191 5-min observation period. The same recorded data were also used to measure thigmotaxis as the 192 total number of squares visited by the fish that were located at the periphery of the walls and the 193 containers (i.e. grey area in Fig. 1) when tested alone.

194

195

Novel object test: Neophobia

196 We divided the test tank into 3 sections by 2 opaque removable partitions that could be moved 197 with pulley system in order to avoid any disturbance (Fig. 2): the first section contained a 198 transparent rectangular container $(20 \times 10 \times 15 \mathrm{~cm})$ where we could confine the audience 199 individuals; the central section contained 2 plastic plants that could be used as a shelter; the end 200 section contained a novel object that consisted of a small colored figurine (around $4 \mathrm{~cm}$ in 201 diameter) placed $1 \mathrm{~cm}$ from the food box. The food box, which was made of a small net and 202 contained brine shrimps, was used as an attractive olfactory signal to encourage the fish to 203 approach the novel object. At the beginning of a trial, we introduced the focal fish into the first 204 section of the tank, and then we gently removed the partitions A and B after 5 and 20 min, 205 respectively (Fig. 2). We let the fish acclimatize for $20 \mathrm{~min}$ before removing partition $\mathrm{B}$ to 206 ensure that no fish would swim erratically and enter in contact with the novel object by chance. 207 Then, we estimated neophobia as the latency to come within $1 \mathrm{~cm}$ of the novel object. A trial 208 ended when the focal fish had approached the food or after $20 \mathrm{~min}$, whichever occurred first. For 209 the audience treatment, 2 males were confined in the container before the focal fish was 210 introduced, while the container was kept empty for the alone treatment. We placed the audience 211 in the first section of the aquarium to maximize the chance of observing differences between the 2122 treatments in the case where some fish would exhibit maximum latencies when tested alone.

214 After the 2 tests were completed, we estimated for each fish its levels of plasticity under 3 215 different social gradients as the difference in absolute and relative values between i) the 2 216 replicates of the alone treatment (e.g. |T1-T2|) (gradient A), ii) the 2 replicates of the audience 217 treatments (e.g. $|\mathrm{T} 3-\mathrm{T} 4|)$ (gradient B), and iii) the mean trait value in the alone treatment and the 
218 mean trait value in the audience treatment (e.g. |(T1+T2-T3+T4)/2|) (gradient C). For each

219 individual, therefore, we had a maximum of 7 absolute or relative measures of plasticity in 220 personality traits (i.e. one for anxiety, 3 for exploration and 3 for neophobia). For statistical 221 analysis, however, differences between two measures with the highest possible value $(n=12)$ 222 were replaced by missing data in order to avoid ceiling effects.

\section{3. Reversal learning}

225 The fish were trained to perform an associative learning task and then were tested for reversal 226 learning, often viewed as a measure of behavioral flexibility by psychologists and neuroscientists 227 (Fellows \& Farah, 2003; Izquierdo et al., 2007; Haluk \& Floresco, 2009; Izquierdo \& Jentsch, 228 2012). The tests were performed in a tank divided with a transparent removable partition into one observation compartment and one choice compartment that had 2 corridors, separated from each other by a porous partition (Fig. 3). Two visual cues of different shapes and colors (i.e. a yellow cross and a blue square of around $4 \times 4 \mathrm{~cm}$ ) were placed at the end of each corridor. Two identical symbols were also placed in front of the corridors, to ensure that the fish could see them

233 from the observation compartment. Prior to the start of the learning tests, following 2 days of 234 food deprivation, the fish were habituated to the apparatus during 3 periods of 30 min each. We 235 insured that they were all able to explore both corridors of the choice compartment to get food.

236 The fish were tested after $24 \mathrm{~h}$ of food deprivation (unless they made no choice in the first $20 \mathrm{~min}$ 237 in which case they were deprived of food for another 24-hour period) until they choose the 238 correct corridor 6 consecutive times. During the test, the position of each cue remained constant 239 from trial to trial, but the rewarded cue, as well as its position, were counterbalanced across 240 subjects. At the beginning of each trial, the focal fish was placed in the observation compartment 241 for 5 min or until it has ceased freezing or swimming erratically. We then placed 3 spirulina 242 flakes on the side of the rewarded cue and gently lifted the removable partition to allow the fish 243 to enter the choice compartment and make a decision. To prevent the food from scattering in the 244 aquarium and from being visible from the observation compartment, it was placed behind a 245 curved PVC partition. We considered that the fish had made a choice once half of its body had 246 entered the choice compartment. If the fish succeeded, it could eat the 3 spirulina flakes before 247 returning to the observation chamber. Otherwise, the observer gently activated the removable 248 partition to encourage the fish to return into the observation compartment. One week later, the 
249 fish were tested for reversal learning after 2 days of food deprivation. To ensure that the fish 250 remembered which cue was rewarded in the acquisition phase, we retested them using the same 251 procedure as above until they choose the correct corridor 6 consecutive times. All individuals 252 were then tested for reversal learning 2 to $2.5 \mathrm{~h}$ after they reached this criterion, except 2 fish that 253 refused to participate in the reversal learning trials and hence were tested again after $24 \mathrm{~h}$. The 254 reversal learning trials were performed in the same manner as described above, except that the 255 previously unrewarded cue was now rewarded, and the previously rewarded cue was 256 unrewarded. Each fish was tested until it chose the correct corridor 6 consecutive times. The 257 number of trials required by each fish to reach this criterion was then used as a proxy of its 258 plasticity.

259

\section{Statistical analyses}

261 Our complete data set contained 5 missing values. In 3 occasions the missing values were due to 262 technical problems. In the 2 other cases, we replaced the anxiety score of a fish by a missing 263 value because it stayed immobile in the middle of the test arena for the entire duration of the test, 264 while another individual was never able to explore the choice compartments of the learning 265 apparatus during the familiarization period and hence had no value for the reversal learning test.

267 To explore how the 9 measures of plasticity were related to each other, we used Spearman rank 268 correlations to test for pairwise correlations and applied the improved Bonferroni correction to 269 account for multiple comparisons (Hochberg, 1988). Correlations were calculated using both 270 absolute and relative measures of plasticity. We also performed a Principal Component Analysis 271 (PCA) on the 9 measures of plasticity in absolute value to test for a general plasticity that would 272 be explained by a single factor. Finally, we tested whether the level of plasticity in exploration 273 and neophobia differed among the social gradients for a given individual with paired-t tests and 274 explored whether the amount of plasticity in personality displayed in response to gradients A and 275 B depended on the level of expression of the trait during the first exposure to the stimuli (i.e. T1 276 or T3) by using Spearman rank correlations.

277 All statistical analyses were conducted on R 3.2.4 (R_Core_Team 2016). PCA was performed 278 on the standardized data and using the dudi.pca function. 


\section{RESULTS}

281 Although individual measures of plasticity in absolute value were positively correlated in the 282 majority (31/36) of pairwise comparisons (Table 1), none of these correlations were significant 283 (Bonferroni corrected $\alpha=0.001$ ). Explicitly, the correlations were at best moderate (i.e. between 2840.4 and 0.59 ) with an average correlation equal to 0.174. The correlations were even smaller 285 when considering the differences in relative value (Table 2) as always at least 21\% (and up to $28650 \%$ ) of the focal fish adjusted their behavior to the changes in social conditions in the opposite 287 direction to that of their congeners. For instance, 21 fish were more exploratory when tested with 288 an audience than alone, but 12 other fish on the contrary had a higher exploration score in the 289 alone treatment. Thus, our findings indicate that an individual's level of plasticity was weakly 290 repeatable across contexts and behaviors. This conclusion is further supported by the fact that 4 291 principal components with eigenvalues greater than one were extracted from the PCA, with each 292 of them explaining only a small portion of the total variance (Table 3). Furthermore, the 4 first 293 principal components had positive and negative loading values (Table 3), which reflects the fact 294 that the different measures of plasticity were not equivalent.

295

296 There were differences in the level of plasticity expressed by individuals depending on the social 297 gradient they were exposed to. Most notably, the magnitude of the changes in behavioral 298 responses was smaller when the fish were tested twice without an audience compared to when 299 they were observed 2 times by different audience individuals or when we compared the average 300 response with and without an audience (Fig. 4). Yet, only the difference between the gradient A 301 and $\mathrm{C}$ was significant for measures of neophobia $\left(\mathrm{t}_{29}=-2.347, \mathrm{P}=0.026\right)$. Finally, for the 3 302 personality traits measured, we found a significant association between the amount of plasticity 303 exhibited and the value of the trait during the first trial (Neophobia, $\mathrm{A}: \mathrm{r}_{\mathrm{s}}=0.644, \mathrm{~N}=30, \mathrm{P}<0.001$; $304 \mathrm{~B}: \mathrm{r}_{\mathrm{s}}=0.526, \mathrm{~N}=26, \mathrm{P}=0.006$; Exploration, $\mathrm{A}: \mathrm{r}_{\mathrm{s}}=0.561, \mathrm{~N}=31, \mathrm{P}=0.001 ; \mathrm{B}: \mathrm{r}_{\mathrm{s}}=0.488, \mathrm{~N}=33$, $305 \mathrm{P}=0.004$ \& Anxiety, $\mathrm{A}: \mathrm{r}_{\mathrm{s}}=0.481, \mathrm{~N}=28, \mathrm{P}=0.010$; see also supplementary material 2). 306 Individuals that exhibited the greatest plasticity, therefore, showed greater expression of the trait 307 when they were exposed to the stimuli for the first time.

308

309 DISCUSSION

310 Using sailfin mollies, we tested whether some individuals are generally more behaviorally plastic 
311 than others, or if an individual's ability to adjust its behavior in response to changing conditions

312 is trait and/or context-dependent. We found that the correlations between pairwise measures of

313 plasticity were non-significant, thus indicating that the most plastic individuals were not the

314 same in all the tests. Although our sample size is small, and the power to detect significant

315 associations is relatively low, this conclusion is supported by the fact that the correlations were

316 only weak or moderate in strength. The correlations were particularly weak when considering not

317 only the magnitude but also the direction of behavioral changes, as individuals differed widely

318 from each other in how they were affected by the treatments. Two non-exclusive explanations

319 could account for our finding.

320

321 First, the lack of correlation between the different measures of plasticity could result from 322 differences among individuals in their internal state that would have affected their willingness to 323 express behavioral plasticity in response to environmental conditions (Wolf \& Weissing, 2010).

324 Indeed, the extent to which an animal actually changes its behavior in response to changing 325 stimuli is a measure of realized plasticity and hence does not necessarily reflect potential 326 plasticity (Stamps \& Krishnan, 2014; Stamps, 2016). Individuals with high potential plasticity, 327 therefore, might have exhibited under certain conditions or for certain traits little plasticity, 328 resulting in a weak association between the different measures of realized plasticity. Our results 329 partly support this hypothesis, as they suggest that individuals would adjust the magnitude of 330 their behavioral responses depending on the benefits of plasticity. Indeed, we found that the fish 331 exhibiting the greatest plasticity in relative value in response to the social gradients A and B 332 showed greater expression of the trait (i.e. were the most neophobic, exploratory and anxious 333 individuals) when they were exposed to the stimuli for the first time, probably because those 334 individuals tend to overestimate the danger when confronted to a novel situation, thereby 335 reacting inappropriately. In accordance to this idea, Mitchell \& Biro (2017) also found that active 336 individuals that deplete energy reserves at a faster rate than less active fish, were more 337 responsive to food deprivation. Nonetheless, individuals that were the most plastic in their 338 activity level in response to changes in food abundance were not the most plastic in response to 339 changes in temperature (Mitchell \& Biro, 2017). The importance of past behavior on the 340 expression of behavioral plasticity, therefore, would depend on the environmental gradient used 341 and/or on the trait being measured. Given the fact that all subjects in our study experienced the 
342 trials in the same order, however, this explanation alone cannot account for the absence of

343 significant correlations among the different measures of plasticity.

344

345 Thus, another non-mutually exclusive interpretation of our findings is that behavioral plasticity is 346 not a general characteristic of individuals, such that individuals expressing high behavioral 347 plasticity in one context could be limited in the amount of plasticity they can express in other 348 contexts. Particularly, different measures of behavioral plasticity taken under different conditions 349 or using different traits, would not be equivalent because they are controlled by different neural, 350 physiological or behavioral mechanisms, so that some behavioral traits might be more 351 constrained than others. Supporting this idea, the PCA analysis revealed that the first component 352 accounted for less than $28 \%$ of the total variance, which is low compared to results that are 353 usually interpreted as support for a general factor (see Shaw \& Schmelz, 2017 and references 354 therein). Our interpretation is also supported by the fact that the rate of reversal learning, which 355 is regularly used as a proxy for behavioral plasticity (e.g. Guillette et al., 2010; Lucon-Xiccato \& 356 Bisazza, 2014; Pintor et al., 2014), was very weakly or not correlated with the other measures of 357 individual plasticity. There is evidence that reversal learning tasks assess distinct neurological 358 abilities from other tasks used to measure behavioral plasticity, such as set-shifting or self359 control tasks (Audet \& Lefebvre, 2017). This might explain why different measures of plasticity 360 are uncorrelated. Accordingly, we found that reversal learning performance loaded negatively on 361 the first principal component contrary to the 8 other measures of plasticity that had positive 362 loadings. Individuals capable of inhibiting a previously rewarded behavior more quickly, 363 therefore, would possess particular skills that would not be associated with greater plasticity in 364 personality. In particular, our results are consistent with the hypothesis that plasticity in 365 personality traits may be restrained by the inability of individuals to detect environmental 366 changes. Indeed, as anticipated, fish exhibited on average greater plasticity between the alone 367 and audience treatments than between the 2 trials of the audience treatment for both personality 368 traits measured (e.g. exploration and neophobia). Furthermore, the correlation between the levels 369 of plasticity in exploration and neophobia was stronger when plasticity was measured as the 370 difference between the 2 trials with a randomly selected audience than between the audience and 371 alone treatments. This finding indicates that individuals were more consistent in their plasticity 372 when the changes in environmental conditions were subtle, as only more neophobic (and less 
373 exploratory) individuals that explore their environment slowly and accurately could then detect 374 and respond to such environmental changes (Sih \& Giudice, 2012). Thus, our results suggest that 375 trade-offs between conflicting selection pressures might maintain intra-individual variation in 376 behavioral plasticity. However, in order to confirm this hypothesis, further studies will be 377 required to demonstrate the adaptive nature of intra-individual differences in the expression of 378 behavioral plasticity. Such studies will consist notably in i) testing the effect of spatial and/or 379 temporal environmental heterogeneity on the repeatability of behavioral plasticity, and ii) 380 measuring the fitness consequences of individual plasticity in various contexts.

381

\section{Conclusions}

383 In conclusion, although we cannot conclude from this study to what extent individuals are 384 limited in their level of behavioral plasticity by neural and/or physiological constraints, our 385 findings contradict the widespread (though largely untested) idea that some individuals would be 386 generally more behaviorally plastic than others (Dingemanse \& Wolf, 2013, but see Audet \& 387 Lefebvre, 2017). Whether behavioral plasticity is a repeatable trait or not at the individual level 388 may have important evolutionary consequences, for instance through affecting population 389 stability or persistence (Wolf \& Weissing, 2012; Sih et al., 2012). Additional studies, therefore, 390 should be conducted to investigate the importance of trade-offs between conflicting selection 391 pressures on the maintenance of intra-individual variation in behavioral plasticity and examine 392 the fitness consequences of behavioral plasticity in different contexts.

393

\section{Acknowledgements}

395 We would like to thank Richard Khoury who designed a custom software, allowing us to conduct 396 more precise observations on the video recordings of sailfin mollies. 


\section{REFERENCES}

399 Audet J-N., Lefebvre L. 2017. What's flexible in behavioral flexibility? Behavioral Ecology

400 28:943-947. DOI: 10.1093/beheco/arx007.

401

402

403

404

405

406

407

408

409

410

411

412

413

414

415

416

417

418

419

420

421

422

423

424

425

426

427

428

Bensky MK., Paitz R., Pereira L., Bell AM. 2017. Testing the predictions of coping styles theory in threespined sticklebacks. Behavioural Processes 136:1-10. DOI: 10.1016/j.beproc.2016.12.011.

Biro PA., Beckmann C., Stamps JA. 2010. Small within-day increases in temperature affects boldness and alters personality in coral reef fish. Proceedings of the Royal Society B: Biological Sciences 277:71-77. DOI: 10.1098/rspb.2009.1346.Brucks D., Marshall-Pescini S., Wallis LJ., Huber L., Range F. 2017. Measures of dogs' inhibitory control abilities do not correlate across tasks. Frontiers in Psychology 8:1-17. DOI:

10.3389/fpsyg.2017.00849.

Carere C., Locurto C. 2011. Interaction between animal personality and animal cognition. Current Zoology 57:491-498. DOI: 10.1093/czoolo/57.4.491.

Chittka L., Skorupski P., Raine NE. 2009. Speed-accuracy tradeoffs in animal decision making. Trends in Ecology \& Evolution 24:400-407. DOI: 10.1016/j.tree.2009.02.010.

DeWitt T., Sih A., Wilson D. 1998. Costs and limits of phenotypic plasticity. Trends in Ecology \& Evolution 13:77-81. DOI: 10.1016/S0169-5347(97)01274-3.

Dingemanse NJ., Wolf M. 2013. Between-individual differences in behavioural plasticity within populations: causes and consequences. Animal Behaviour 85:1031-1039. DOI: 10.1016/j.anbehav.2012.12.032.

Dubois F., Morand-Ferron J., Giraldeau L-A. 2010. Learning in a game context: strategy choice by some keeps learning from evolving in others. Proceedings of the Royal Society B: Biological Sciences 277:3609-3616. DOI: 10.1098/rspb.2010.0857.

Fellows LK., Farah MJ. 2003. Ventromedial frontal cortex mediates affective shifting in humans: evidence from a reversal learning paradigm. Brain : a journal of neurology 126:1830-7. DOI: $10.1093 /$ brain/awg 180 .

Fraser B a., Janowitz I., Thairu M., Travis J., Hughes K a. 2014. Phenotypic and genomic plasticity of alternative male reproductive tactics in sailfin mollies. Proceedings of the Royal Society B: Biological Sciences 281:20132310-20132310. DOI:

10.1098/rspb.2013.2310. 
429 Gabriel W., Luttbeg B., Sih A., Tollrian R. 2005. Environmental tolerance, heterogeneity, and 430 the evolution of reversible plastic responses. The American Naturalist 166:339-353. DOI: 10.1086/432558.

432

433

434

435

436

437

438

439

440

441

442

443

444

445

446

447

448

449

450

451

452

453

454

455

456

457

458

459

Gibelli J., Dubois F. 2017. Does personality affect the ability of individuals to track and respond to changing conditions? Behavioral Ecology 28:101-107. DOI: 10.1093/beheco/arw137.

Glorfeld LW. 1995. An Improvement on Horn's Parallel Analysis Methodology for Selecting the Correct Number of Factors to Retain. Educational and Psychological Measurement 55:377393. DOI: $10.1177 / 0013164495055003002$.

Guayasamin OL., Couzin ID., Miller NY. 2017. Behavioural plasticity across social contexts is regulated by the directionality of inter-individual differences. Behavioural Processes 141:196-204. DOI: 10.1016/j.beproc.2016.10.004.

Guido JM., Biondi LM., Vasallo AI., Muzio RN. 2017. Neophobia is negatively related to reversal learning ability in females of a generalist bird of prey, the Chimango Caracara, Milvago chimango. Animal Cognition 20:591-602. DOI: 10.1007/s10071-017-1083-9.

Guillette LM., Reddon AR., Hoeschele M., Sturdy CB. 2010. Sometimes slower is better: slowexploring birds are more sensitive to changes in a vocal discrimination task. Proceedings of the Royal Society B: Biological Sciences 278:767-773. DOI: 10.1098/rspb.2010.1669.

Haluk DM., Floresco SB. 2009. Ventral striatal dopamine modulation of different forms of behavioral flexibility. Neuropsychopharmacology : official publication of the American College of Neuropsychopharmacology 34:2041-52. DOI: 10.1038/npp.2009.21.

Hau M., Goymann W. 2015. Endocrine mechanisms, behavioral phenotypes and plasticity: known relationships and open questions. Frontiers in Zoology 12:S7. DOI: 10.1186/17429994-12-S1-S7.

Hochberg Y. 1988. A Sharper Bonferroni Procedure for Multiple Tests of Significance. Biometrika 75:800. DOI: 10.2307/2336325.

Izquierdo A., Newman TK., Higley JD., Murray E a. 2007. Genetic modulation of cognitive flexibility and socioemotional behavior in rhesus monkeys. Proceedings of the National Academy of Sciences of the United States of America 104:14128-33. DOI: 10.1073/pnas.0706583104.

Izquierdo A., Jentsch JD. 2012. Reversal learning as a measure of impulsive and compulsive behavior in addictions. Psychopharmacology 219:607-20. DOI: 10.1007/s00213-011-2579- 
460

461

462

463

464

465

466

467

468

469

470

471

472

473

474

475

476

477

478

479

480

481

482

483

484

485

486

487

488

489

490

7.

Johnson-Ulrich L., Johnson-Ulrich Z., Holekamp K. 2018. Proactive behavior, but not inhibitory control, predicts repeated innovation by spotted hyenas tested with a multi-access box. Animal Cognition 21:379-392. DOI: 10.1007/s10071-018-1174-2.

Logan CJ. 2016. Behavioral flexibility and problem solving in an invasive bird. PeerJ 4:e1975. DOI: $10.7717 /$ peerj.1975.

Logan CJ. 2016. Behavioral flexibility in an invasive bird is independent of other behaviors. PeerJ 4:e2215. DOI: 10.7717/peerj.2215.

Lucon-Xiccato T., Bisazza A. 2014. Discrimination reversal learning reveals greater female behavioural flexibility in guppies. Biology Letters 10:20140206-20140206. DOI: 10.1098/rsbl.2014.0206.

Mamuneas D., Spence a J., Manica A., King AJ. 2015. Bolder stickleback fish make faster decisions, but they are not less accurate. Behavioral Ecology 26:91-96. DOI: 10.1093/beheco/aru160.

Mathot KJ., Wright J., Kempenaers B., Dingemanse NJ. 2012. Adaptive strategies for managing uncertainty may explain personality-related differences in behavioural plasticity. Oikos 121:1009-1020. DOI: 10.1111/j.1600-0706.2012.20339.x.

Maximino, C., de Brito, T. M., da Silva Batista, A. W., Herculano, A. M., Morato, S., \& Gouveia, A. 2010. Measuring anxiety in zebrafish: A critical review. Behavioural Brain Research, 214:157-171. DOI: 10.1016/j.bbr.2010.05.031

Mazza V., Eccard JA., Zaccaroni M., Jacob J., Dammhahn M. 2018. The fast and the flexible: cognitive style drives individual variation in cognition in a small mammal. Animal Behaviour 137:119-132. DOI: 10.1016/j.anbehav.2018.01.011.

McGlothlin JW., Jawor JM., Ketterson ED. 2007. Natural Variation in a Testosterone-Mediated Trade-Off between Mating Effort and Parental Effort. The American Naturalist 170:864875. DOI: $10.1086 / 522838$.

Mitchell DJ., Biro PA. 2017. Is behavioural plasticity consistent across different environmental gradients and through time? Proceedings of the Royal Society B: Biological Sciences 284:20170893. DOI: 10.1098/rspb.2017.0893.

Montiglio PO., Wey TW., Chang AT., Fogarty S., Sih A. 2017. Correlational selection on personality and social plasticity: morphology and social context determine behavioural 
491

492

493

494

495

496

497

498

499

500

501

502

503

504

505

506

507

508

509

510

511

512

513

514

515

516

517

518

519

520 DOI: $10.3389 /$ fevo.2014.00069.

521 Toscano BJ. 2017. Prey behavioural reaction norms: response to threat predicts susceptibility to

effects on mating success. Journal of Animal Ecology 86:213-226. DOI: 10.1111/13652656.12610 .

Morand-Ferron J., Varennes E., Giraldeau L-A. 2011. Individual differences in plasticity and sampling when playing behavioural games. Proceedings of the Royal Society B: Biological Sciences 278:1223-1230. DOI: 10.1098/rspb.2010.1769.

Pintor LM., McGhee KE., Roche DP., Bell AM. 2014. Individual variation in foraging behavior reveals a trade-off between flexibility and performance of a top predator. Behavioral Ecology and Sociobiology 68:1711-1722. DOI: 10.1007/s00265-014-1779-7.

Réale D., Reader SM., Sol D., McDougall PT., Dingemanse NJ. 2007. Integrating animal temperament within ecology and evolution. Biological Reviews 82:291-318. DOI: 10.1111/j.1469-185X.2007.00010.x.

Shaw RC., Schmelz M. 2017. Cognitive test batteries in animal cognition research: evaluating the past, present and future of comparative psychometrics. Animal Cognition 20:10031018. DOI: $10.1007 / \mathrm{s} 10071-017-1135-1$.

Sih A., Bell AM. 2008. Insights for Behavioral Ecology from Behavioral Syndromes. Advances in the study of behavior 38:227-281. DOI: 10.1016/S0065-3454(08)00005-3.

Sih A., Del Giudice M. 2012. Linking behavioural syndromes and cognition: a behavioural ecology perspective. Philosophical transactions of the Royal Society of London. Series B, Biological sciences 367:2762-72. DOI: 10.1098/rstb.2012.0216.

Sih A., Cote J., Evans M., Fogarty S., Pruitt J. 2012. Ecological implications of behavioural syndromes. Ecology Letters 15:278-289. DOI: 10.1111/j.1461-0248.2011.01731.x.

Sol D., Lefebvre L. 2000. Behavioural flexibility predicts invasion success in birds introduced to New Zealand. Oikos 90:599-605. DOI: 10.1034/j.1600-0706.2000.900317.x.

Sol D., Timmermans S., Lefebvre L. 2002. Behavioural flexibility and invasion success in birds. Animal Behaviour 63:495-502. DOI: 10.1006/anbe.2001.1953.

Stamps JA. 2016. Individual differences in behavioural plasticities. Biological Reviews 91:534567. DOI: $10.1111 /$ brv.12186.

Stamps JA., Krishnan V V. 2014. Individual differences in the potential and realized

19 developmental plasticity of personality traits. Frontiers in Ecology and Evolution 2:1-15. 
522 predation. Animal Behaviour 132:147-153. DOI: 10.1016/j.anbehav.2017.08.014.

523 Travis J., Woodward BD. 1989. Social context and courtship flexibility in male sailfin mollies,

524 Poecilia latipinna (Pisces: Poecillidae). Animal Behaviour 38:1001-1011. DOI:

$525 \quad$ 10.1016/S0003-3472(89)80139-3.

526 Wolf M., van Doorn GS., Weissing FJ. 2008. Evolutionary emergence of responsive and

527 unresponsive personalities. Proceedings of the National Academy of Sciences 105:15825-

528 15830. DOI: 10.1073/pnas.0805473105.

529 Wolf M., Weissing FJ. 2010. An explanatory framework for adaptive personality differences.

530 Philosophical transactions of the Royal Society of London. Series B, Biological sciences

531 365:3959-3968. DOI: 10.1098/rstb.2010.0215.

532 Wolf M., Van Doorn GS., Weissing FJ. 2011. On the coevolution of social responsiveness and

533 behavioural consistency. Proceedings of the Royal Society B: Biological Sciences 278:440-

534 448. DOI: $10.1098 / \mathrm{rspb} .2010 .1051$.

535 Wolf M., Weissing FJ. 2012. Animal personalities: Consequences for ecology and evolution.

536 Trends in Ecology and Evolution 27:452-461. DOI: 10.1016/j.tree.2012.05.00 
Figure 1

Top view of the tank used to measure exploration and anxiety.

The 4 containers, placed in each corner of the tank, housed a single individual when the fish was tested with an audience or were kept empty otherwise. The grey area delimits the area considered to measure anxiety in the alone treatment.

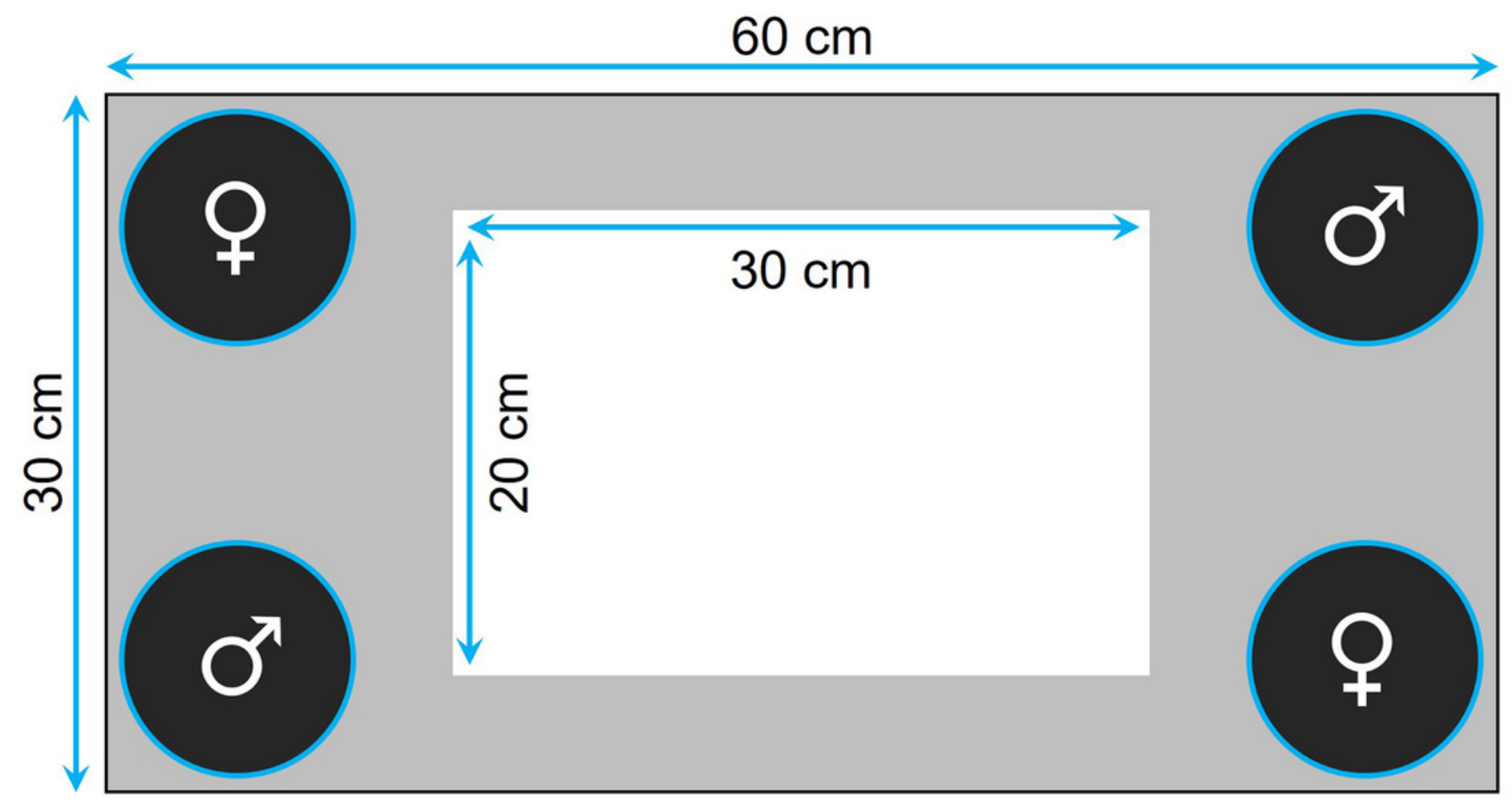


Figure 2

Top view of the tank used to measure neophobia.

The tank was divided into 3 identical sections $(30 \times 20 \mathrm{~cm})$ by two opaque removable partitions (blue dotted lines). Section 1 contained a transparent box used to confine the audience males, section 2 was equipped with two plastic plants that could be used as a shelter and section 3 contained a novel object and a food box.

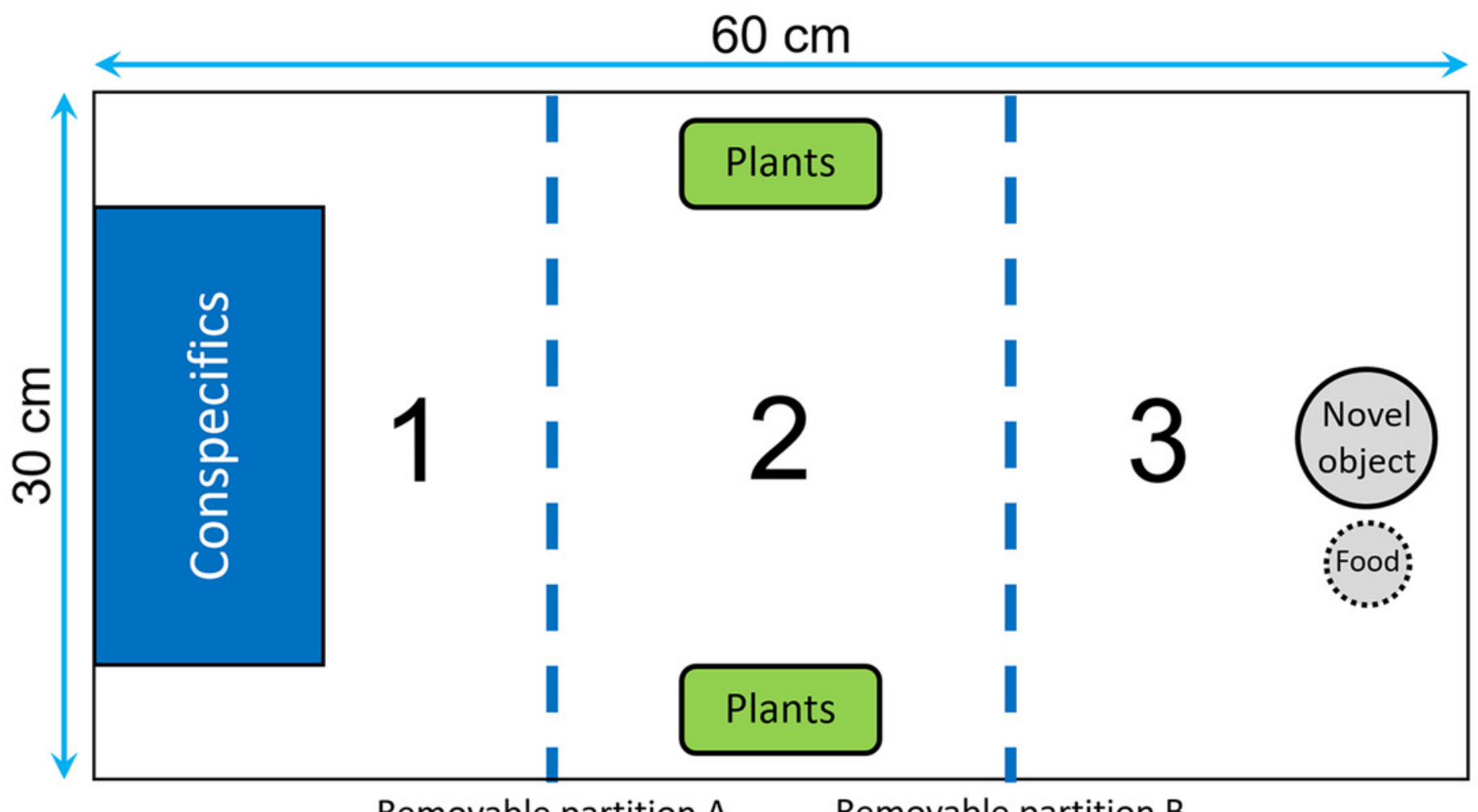

Removable partition A Removable partition B 


\section{Figure 3}

Top view of the tank used to measure reversal learning speed.

The tank was divided in 2 sections $(30 \times 30 \mathrm{~cm})$ by a transparent removable partition (blue dotted line): an observation compartment and a choice compartment divided into 2 corridors Two visual cues (a blue square and a yellow cross of around $4 \times 4 \mathrm{~cm}$ ) were placed both in front and at the end of each corridor to ensure that the fish could see them from the observation compartment and learn which cue was rewarded.

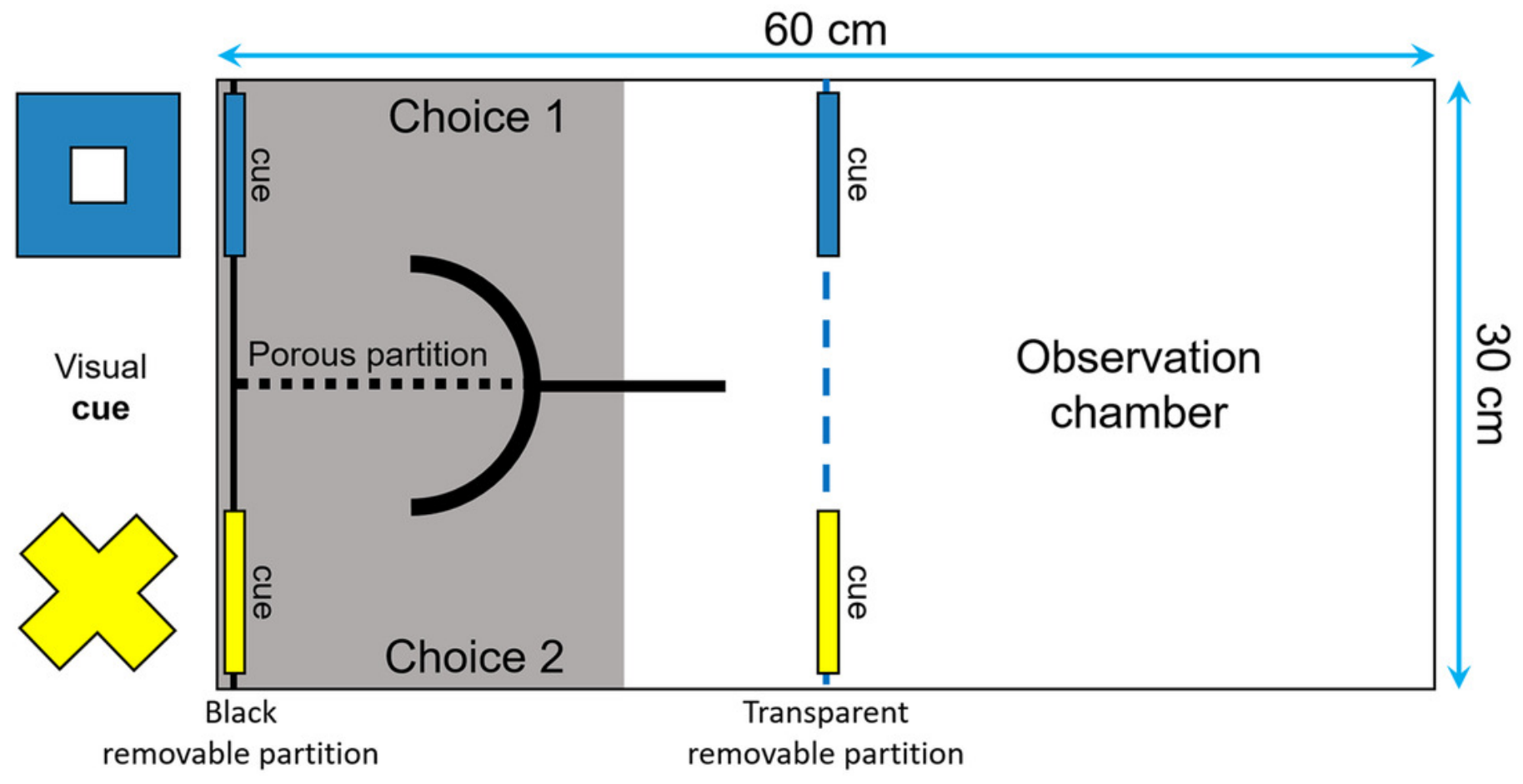


Figure 4

Mean ( \pm SEM) behavioral plasticity in neophobia (panel $A$ ) and exploration (panel $B$ ) measured under 3 different social gradients.

Behavioral plasticity was estimated as the difference in absolute value between i) the 2 replicates of the alone treatment (gradient A), ii) the 2 replicates of the audience treatment (gradient B), and iii) the mean trait value in the alone treatment and the mean trait value in the audience treatment (gradient $\mathrm{C}$ ).
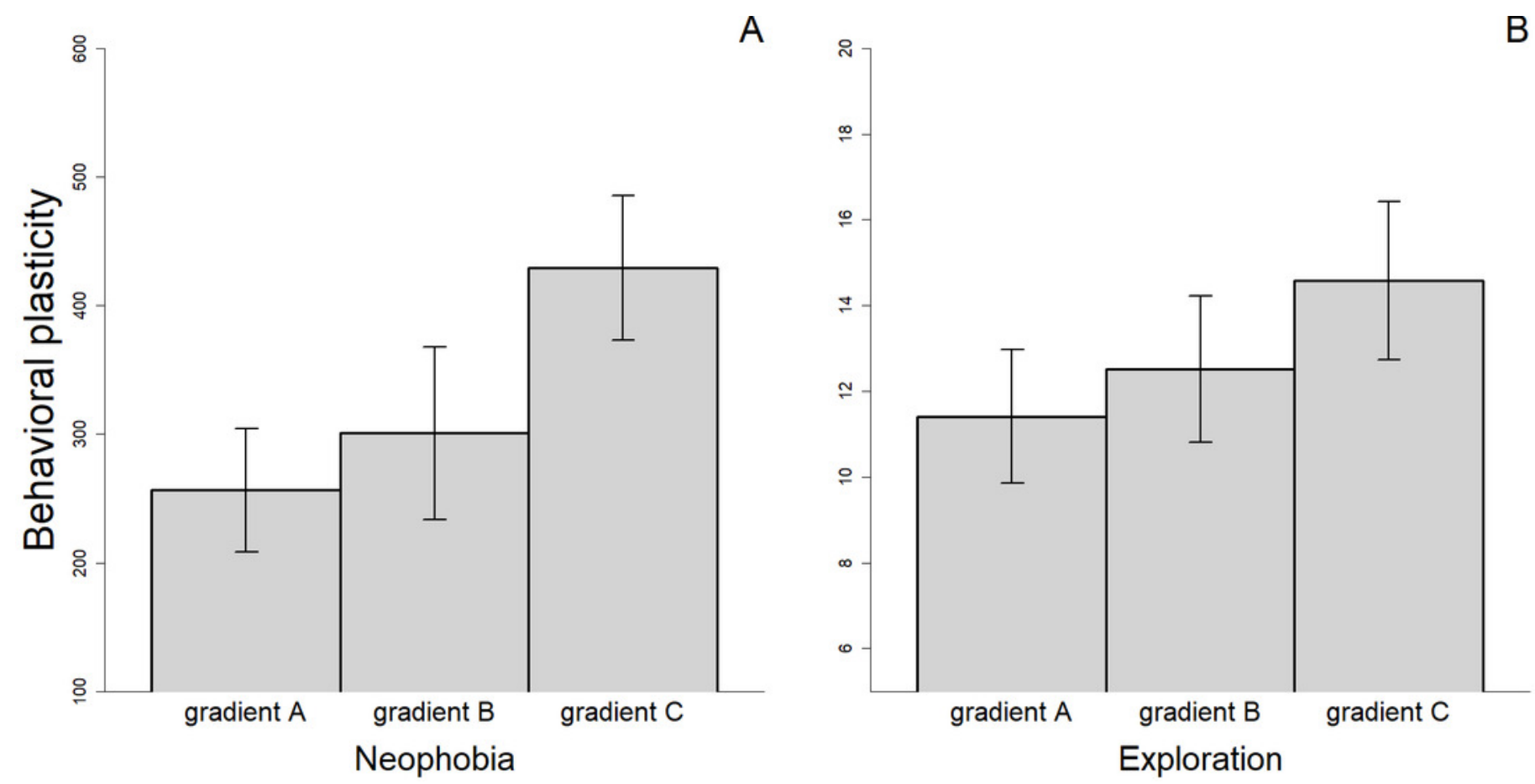


\section{Table $\mathbf{1}$ (on next page)}

Pairwise Spearman rank correlations among the 9 measures of behavioral plasticity estimated as the differences in absolute value.

Reversal learning scores (i.e. the number of trials to reach the learning criterion in the reversal task) were multiplied by -1 in such a way that the subjects that were faster to reverse had the highest scores. 


\begin{tabular}{|c|c|c|c|c|c|c|c|c|c|}
\hline & & $\begin{array}{c}\text { Neophobia } \\
\text { A }\end{array}$ & $\begin{array}{c}\text { Exploration } \\
\text { A }\end{array}$ & $\begin{array}{c}\text { Anxiety } \\
\text { A }\end{array}$ & $\begin{array}{c}\text { Neophobia } \\
\text { B }\end{array}$ & $\begin{array}{c}\text { Exploration } \\
\text { B }\end{array}$ & $\begin{array}{c}\text { Neophobia } \\
\text { C }\end{array}$ & $\begin{array}{l}\text { Exploration } \\
\text { C }\end{array}$ & $\begin{array}{l}\text { Mating } \\
\text { behavior }\end{array}$ \\
\hline \multirow{3}{*}{ Exploration A } & $r_{s}$ & 0.369 & & & & & & & \\
\hline & $\mathrm{P}$ & 0.053 & & & & & & & \\
\hline & $\mathrm{N}$ & 28 & & & & & & & \\
\hline \multirow{3}{*}{ Anxiety A } & $r_{s}$ & 0.530 & 0.281 & & & & & & \\
\hline & $\mathrm{P}$ & 0.004 & 0.148 & & & & & & \\
\hline & $\mathrm{N}$ & 27 & 28 & & & & & & \\
\hline \multirow{3}{*}{ Neophobia B } & $r_{s}$ & 0.259 & 0.309 & -0.217 & & & & & \\
\hline & $\mathrm{P}$ & 0.221 & 0.142 & 0.319 & & & & & \\
\hline & $\mathrm{N}$ & 24 & 24 & 23 & & & & & \\
\hline \multirow{3}{*}{ Exploration B } & $r_{s}$ & 0.171 & 0.277 & -0.115 & 0.429 & & & & \\
\hline & $\mathrm{P}$ & 0.366 & 0.132 & 0.560 & 0.029 & & & & \\
\hline & $\mathrm{N}$ & 30 & 31 & 28 & 26 & & & & \\
\hline \multirow{3}{*}{ Neophobia C } & $r_{s}$ & 0.168 & 0.053 & 0.083 & 0.508 & 0.177 & & & \\
\hline & $\mathrm{P}$ & 0.374 & 0.780 & 0.676 & 0.008 & 0.332 & & & \\
\hline & $\mathrm{N}$ & 30 & 30 & 28 & 26 & 32 & & & \\
\hline \multirow{3}{*}{ Exploration C } & $r_{s}$ & 0.348 & 0.231 & 0.294 & 0.417 & -0.285 & 0.214 & & \\
\hline & $\mathrm{P}$ & 0.059 & 0.212 & 0.129 & 0.034 & 0.108 & 0.239 & & \\
\hline & $\mathrm{N}$ & 30 & 31 & 28 & 26 & 33 & 32 & & \\
\hline \multirow{3}{*}{$\begin{array}{c}\text { Mating } \\
\text { behavior }\end{array}$} & $r_{s}$ & 0.444 & 0.178 & 0.007 & 0.095 & 0.033 & 0.254 & 0.080 & \\
\hline & $P$ & 0.014 & 0.339 & 0.974 & 0.645 & 0.856 & 0.160 & 0.657 & \\
\hline & $\mathrm{N}$ & 30 & 31 & 28 & 26 & 33 & 32 & 33 & \\
\hline \multirow{3}{*}{$\begin{array}{l}\text { Reversal } \\
\text { learning }\end{array}$} & $r_{s}$ & 0.019 & 0.305 & 0.386 & 0.089 & 0.041 & -0.047 & 0.154 & -0.285 \\
\hline & $P$ & 0.922 & 0.101 & 0.043 & 0.667 & 0.824 & 0.798 & 0.401 & 0.113 \\
\hline & $\mathrm{N}$ & 30 & 30 & 28 & 26 & 32 & 32 & 32 & 32 \\
\hline
\end{tabular}




\section{Table 2 (on next page)}

Pairwise Spearman rank correlations among the 9 measures of behavioral plasticity estimated as the differences in relative value.

Reversal learning scores (i.e. the number of trials to reach the learning criterion in the reversal task) were multiplied by -1 in such a way that the subjects that were faster to reverse had the highest scores. 
1

\begin{tabular}{|c|c|c|c|c|c|c|c|c|c|}
\hline & & $\begin{array}{c}\text { Neophobia } \\
\text { A }\end{array}$ & $\begin{array}{c}\text { Exploration } \\
\text { A }\end{array}$ & $\begin{array}{c}\text { Anxiety } \\
\text { A }\end{array}$ & $\begin{array}{c}\text { Neophobia } \\
\text { B }\end{array}$ & $\begin{array}{c}\text { Exploration } \\
\text { B }\end{array}$ & $\begin{array}{c}\text { Neophobia } \\
\text { C }\end{array}$ & $\begin{array}{c}\text { Exploration } \\
\text { C }\end{array}$ & $\begin{array}{l}\text { Mating } \\
\text { behavior }\end{array}$ \\
\hline \multirow{3}{*}{ Exploration A } & $r_{s}$ & -0.221 & & & & & & & \\
\hline & $P$ & 0.258 & & & & & & & \\
\hline & $\mathrm{N}$ & 28 & & & & & & & \\
\hline \multirow{3}{*}{ Anxiety A } & $r_{\mathrm{s}}$ & -0.005 & -0.098 & & & & & & \\
\hline & $\mathrm{P}$ & 0.981 & 0.620 & & & & & & \\
\hline & $\mathrm{N}$ & 27 & 28 & & & & & & \\
\hline \multirow{3}{*}{ Neophobia B } & $r_{s}$ & 0.003 & -0.391 & 0.188 & & & & & \\
\hline & $\mathrm{P}$ & 0.987 & 0.059 & 0.391 & & & & & \\
\hline & $\mathrm{N}$ & 24 & 24 & 23 & & & & & \\
\hline \multirow{3}{*}{ Exploration B } & $r_{s}$ & -0.165 & 0.174 & -0.328 & -0.189 & & & & \\
\hline & $P$ & 0.384 & 0.350 & 0.089 & 0.355 & & & & \\
\hline & $\mathrm{N}$ & 30 & 31 & 28 & 26 & & & & \\
\hline \multirow{3}{*}{ Neophobia C } & $r_{s}$ & 0.000 & 0.260 & -0.140 & -0.018 & 0.312 & & & \\
\hline & $\mathrm{P}$ & 0.999 & 0.166 & 0.479 & 0.930 & 0.082 & & & \\
\hline & $\mathrm{N}$ & 30 & 30 & 28 & 26 & 32 & & & \\
\hline \multirow{3}{*}{ Exploration C } & $r_{s}$ & 0.189 & -0.106 & -0.269 & 0.166 & -0.160 & -0.02 & & \\
\hline & $P$ & 0.318 & 0.572 & 0.166 & 0.416 & 0.372 & 0.914 & & \\
\hline & $\mathrm{N}$ & 30 & 31 & 28 & 26 & 33 & 32 & & \\
\hline \multirow{3}{*}{$\begin{array}{l}\text { Mating } \\
\text { behavior }\end{array}$} & $r_{s}$ & -0.275 & -0.167 & -0.035 & 0.115 & 0.114 & 0.312 & -0.095 & \\
\hline & $\mathrm{P}$ & 0.141 & 0.368 & 0.858 & 0.576 & 0.527 & 0.082 & 0.598 & \\
\hline & $\mathrm{N}$ & 30 & 31 & 28 & 26 & 33 & 32 & 33 & \\
\hline \multirow{3}{*}{$\begin{array}{l}\text { Reversal } \\
\text { learning }\end{array}$} & $r_{s}$ & 0.397 & -0.103 & -0.032 & -0.275 & -0.099 & 0.088 & -0.325 & 0.328 \\
\hline & $P$ & 0.030 & 0.588 & 0.870 & 0.174 & 0.591 & 0.631 & 0.069 & 0.067 \\
\hline & $\mathrm{N}$ & 30 & 30 & 28 & 26 & 32 & 32 & 32 & 32 \\
\hline
\end{tabular}

3 


\section{Table 3 (on next page)}

Loadings on the 4 extracted factors. 


\begin{tabular}{lrrrr} 
Plasticity measure & PC1 & PC2 & PC3 & PC4 \\
\hline Neophobia A & 0.705 & 0.502 & -0.298 & 0.043 \\
Exploration A & 0.710 & 0.337 & -0.035 & -0.251 \\
Anxiety A & 0.235 & 0.689 & 0.313 & -0.005 \\
Neophobia B & 0.477 & -0.546 & 0.551 & 0.122 \\
Exploration B & 0.498 & -0.021 & -0.026 & 0.807 \\
Neophobia C & 0.514 & -0.455 & 0.110 & 0.129 \\
Exploration C & 0.606 & -0.241 & 0.452 & -0.467 \\
Mating behavior & 0.568 & -0.129 & -0.704 & -0.157 \\
Reversal learning & -0.043 & 0.716 & 0.439 & 0.105 \\
Eigenvalues & 2.487 & 1.934 & 1.397 & 1.002 \\
\% of variance & $27.6 \%$ & $21.5 \%$ & $15.5 \%$ & $11.1 \%$ \\
\hline
\end{tabular}

